\title{
Identification and characterization of porcine parvovirus in Hunan province, China1)
}

\author{
DUN ZHAO, MEIJUN YAN, XINGLONG YU
}

\author{
College of Veterinary Medicine, Hunan Agricultural University, Changsha, Hunan 410128, China
}

\section{Zhao D., Yan M., Yu X. \\ Identification and characterization of porcine parvovirus in Hunan province, China}

Summary

Porcine parvovirus (PPV) is considered one of the most important infectious agents of reproductive failure in sows. Little information, however, is available on its prevalence in healthy fattening pigs. Therefore, in the present study, 197 fecal swabs, 197 nasal swabs, 389 serum samples, and 310 lung samples were collected from pigs aged 10-25 weeks across Hunan, China, and tested for the presence of PPV. PPV DNA was amplified by polymerase chain reaction, demonstrating an overall positivity rate of $7.69 \%$, with a particularly high infection rate of $22.90 \%$ in the lungs. A total of five PPV strains (PPV-HuN1-5) were isolated on the basis of cytopathic effects in swine testicular cells, and the near-complete genomes were sequenced and subjected to phylogenetic analysis with reference to reported PPV sequences in GenBank. The five Hunan isolates showed a close relationship with each other and predominantly with reported European PPV strains. Moreover, seven amino acid substitutions were detected within the coding region of the VP2 of PPV-HuNs when compared with that of the Chinese vaccine strain PPV-NJ. The relatively high prevalence of PPV discovered in healthy fattening pigs despite a long-term vaccination program in China, highlights the need for improved prevention, monitoring, and control of related diseases in herds.

Keywords: porcine parvovirus, prevalence, fattening pigs, phylogeny

Taxonomically, Ungulate protoparvovirus 1, also known as Porcine parvovirus (PPV), belongs to the genus Protoparvovirus of the subfamily Parvovirinae within the family Parvoviridae (5). The PPV genome consists of a negative-sense single-stranded DNA molecule of about $5 \mathrm{~kb}$ in size. Both terminals of the virus genome form complex palindromic hairpin structures in a Y- or T-like shape (22). The PPV genome comprises two major open reading frames (ORFs), encoding seven proteins by the complementary strand: ORF1 and ORF2 encode non-structural proteins (NS1, NS2, and NS3) and structural proteins (VP1, VP2, and VP3), respectively (20). Furthermore, a late non-structural protein (SAT) is expressed from the same VP2 mRNA, initiated at the seven nucleotides downstream of the VP2 start codon (25).

PPV was first isolated in Germany in 1965 as a contaminant of porcine primary cell cultures (13). Since then, PPV has been identified worldwide, including in Africa, Asia, Australia, Europe, and North America $(1,4,6,17,19)$. In most cases, PPV infection alone

1) This work was supported by the National Key Research and Development Program of China (2017YFD0500104). does not cause clinical symptoms in non-pregnant adult pigs or piglets. However, virulent strains can cause reproductive failure in swine herds, and can lead to clinical still birth, mummification, embryonic death, and infertility (SMEDI) syndrome (14). Despite the wide use of PPV vaccines in China, PPV has remained a constant problem in the pig industry, and several studies reported continuous outbreaks of PPV in pig populations $(8,19)$. However, the general prevalence of PPV is still not well understood in most parts of China. Therefore, the present study was conducted to characterize PPV and to determine its overall prevalence in apparently healthy domestic pigs throughout the Hunan province, China.

\section{Material and methods}

Sample collection. A total of 1093 samples were collected from pig farms and slaughterhouses across the Hunan province from January to August 2017, including 197 fecal swabs, 197 nasal swabs, 389 serum samples, and 310 lung samples. To avoid possible cross-contamination, the samples were collected with sterile instruments and on different days whenever possible. The fecal swabs, nasal swabs, and serum samples were obtained from nine pig farms (Tab. 1), 
Tab. 1. Detailed information on samples and prevalence of PPV in different farms

\begin{tabular}{|c|c|c|c|c|}
\hline \multirow{2}{*}{ Farm } & \multirow{2}{*}{$\begin{array}{l}\text { No. of } \\
\text { samples }\end{array}$} & Fecal swabs & Nasal swabs & Serum \\
\hline & & \multicolumn{3}{|c|}{ PPV-positivity rate (\%) } \\
\hline 1 & 87 & $3.48(1 / 29)$ & $6.90(2 / 29)$ & $6.90(2 / 29)$ \\
\hline 2 & 144 & $2.08(1 / 48)$ & $0(0 / 48)$ & $0(0 / 48)$ \\
\hline 3 & 72 & $4.17(1 / 24)$ & $4.17(1 / 24)$ & $0(0 / 24)$ \\
\hline 4 & 144 & $4.17(2 / 48)$ & $2.08(1 / 48)$ & $0(0 / 48)$ \\
\hline 5 & 144 & $0(0 / 48)$ & $0(0 / 48)$ & $0(0 / 48)$ \\
\hline 6 & 48 & - & - & $0(0 / 48)$ \\
\hline 7 & 32 & - & - & $0(0 / 32)$ \\
\hline 8 & 48 & - & - & $0(0 / 48)$ \\
\hline 9 & 64 & - & - & $3.13(2 / 64)$ \\
\hline Overall & 783 & $2.54(5 / 197)$ & $2.03(4 / 197)$ & $1.03(4 / 389)$ \\
\hline
\end{tabular}

Explanation: - Not identified

Tab. 2. Primers for PPV detection and ORFs gene amplification

\begin{tabular}{|c|c|c|c|}
\hline Primers & Oligonucleotide sequence (5'-3') & Positiona $^{\mathrm{a}}$ & $\begin{array}{c}\text { Annealing } \\
\text { temperature/ } /{ }^{\circ} \mathrm{C}\end{array}$ \\
\hline PPV-F1' & АСTCTCAGCTACTGCAGCAT & 273 & \multirow{2}{*}{55} \\
\hline PPV-R1' & TGCATTATTAACСАTСTACTCСАT & 775 & \\
\hline PPV-F2 & GTGGAAAAGGCTTACAACAA & 692 & \multirow{2}{*}{55} \\
\hline PPV-R2 & GGTTTTGCCTTTTCAAGTATTA & 1337 & \\
\hline PPV-F3 & TAGAAATGATGGCTCAAACC & 1220 & \multirow{2}{*}{55} \\
\hline PPV-R3 & GCTGCTGAGAAGTAGAAGTA & 2544 & \\
\hline PPV-F4 & AAAAGAGCAAGAGGTAAGGG & 2302 & \multirow{2}{*}{55} \\
\hline PPV-R4 & TGACCAAGGTGTTACCATTT & 3139 & \\
\hline PPV-F5 & CGCATCAAGACTCATACATC & 3004 & \multirow{2}{*}{55} \\
\hline PPV-R5 & GTCAGCATTGAAATCATCTGTTAG & 4336 & \\
\hline PPV-F6 & ACAGCACTAAACAATACTGCACCT & 4161 & \multirow{2}{*}{59} \\
\hline PPV-R6 & CTTGGTATAAGTTGTGAATATTCTG & 4539 & \\
\hline
\end{tabular}

Explanation: a - Location of primers relative to the complete genomic sequence of PPV/NADL-2 (GenBank \# NC001718); $\mathrm{b}-\mathrm{F}$ : forward primer; $\mathrm{c}-\mathrm{R}$ : reverse primer

whereas the lung samples were collected from a slaughterhouse in the city of Changsha in the Hunan province. All of the samples came from healthy pigs without apparent clinical signs. The age of the pigs sampled ranged from 10 to 25 weeks. The samples were shipped on ice and kept in a freezer at $-80^{\circ} \mathrm{C}$ until use.

Viral DNA extraction and polymerase chain reaction (PCR) for detecting PPV. After re-suspending the fecal and nasal swabs in $0.5 \mathrm{ml}$ of sterile phosphate-buffered saline (PBS), they were centrifuged at 7,000 $\times \mathrm{g}$ for $5 \mathrm{~min}$, and $0.2 \mathrm{ml}$ of the suspension was transferred into a $2-\mathrm{ml}$ tube that was used for viral DNA extraction. The serum samples were centrifuged at $5,000 \times g$ for $3 \mathrm{~min}$, and $0.2 \mathrm{ml}$ of the suspension was transferred and used for viral DNA extraction. For tissue samples, $0.5 \mathrm{~g}$ aliquots were placed into $2 \mathrm{ml}$ Eppendorf tubes containing $1 \mathrm{ml}$ of sterile PBS with four $1.5 \mathrm{~mm}$ steel balls, and then ground into powder with a Mixer Mill MM 400 (Retsch, Germany) for $3 \mathrm{~min}$. The samples were then clarified by centrifugation at $12,000 \times g$ for $10 \mathrm{~min}$. DNA was extracted according to the manufacturer's instructions (Tiandz Inc., Beijing, China), and the
Tab. 3. Porcine parvovirus reference strains used in the study

\begin{tabular}{|c|c|c|c|c|}
\hline Virus isolate & $\begin{array}{c}\text { GenBank } \\
\text { accession No. }\end{array}$ & $\begin{array}{c}\text { Geographic } \\
\text { origin }\end{array}$ & Dataset & $\begin{array}{c}\text { Collection } \\
\text { date }\end{array}$ \\
\hline 32350005_3h & GQ884039 & Europe & ORFs & 2005 \\
\hline 32260005erh_1h & GQ884036 & Europe & ORFs & 2005 \\
\hline $1242 / 2012$ & MH558678 & Spain & ORFs & 2012 \\
\hline $14 a$ & JN400518 & Germany & ORFs & 2009 \\
\hline $8 a$ & JN400517 & Germany & ORFs & 2009 \\
\hline $7 a$ & JN400516 & Germany & ORFs & 2009 \\
\hline 33800005_1g & GQ884044 & Europe & ORFs & 2006 \\
\hline JY & HM627652 & China & ORFs & 2010 \\
\hline 32260005_1d & GQ884037 & Europe & ORFs & 2005 \\
\hline 32340005_2f & GQ884038 & Europe & ORFs & 2005 \\
\hline 33760005_3a & GQ884041 & Europe & ORFs & 2006 \\
\hline $693 a$ & JN400519 & Austria & ORFs & 2009 \\
\hline 33780005_2g & GQ884042 & Europe & ORFs & 2006 \\
\hline Challenge & AY684866 & United Kingdom & ORFs & 1986 \\
\hline Kresse & U44978 & USA & ORFs & 1985 \\
\hline 40570005_4e & GQ884047 & USA & ORFs & 2006 \\
\hline HN-2011 & JX992846 & China & ORFs & 2008 \\
\hline ZJ & EU790642 & China & ORFs & 2004 \\
\hline HNLY201301 & MF447833 & China & ORFs & 2013 \\
\hline NADL-2 & L23427 & USA & ORFs & 1976 \\
\hline YL & JN860197 & China & ORFs & - \\
\hline T142_Korea & KY994646 & South Korea & ORFs & 2016 \\
\hline LZ & HM627653 & China & ORFs & 2009 \\
\hline BQ & EU790641 & China & ORFs & 2006 \\
\hline $\mathrm{HN}-\mathrm{H}$ & KF429253 & China & ORFs & 2012 \\
\hline PPV2010 & JN872448 & China & ORFs & 2010 \\
\hline SR-1 & DQ675456 & China & ORFs & - \\
\hline GD2013 & KX242359 & China & ORFs & 2013 \\
\hline $\mathrm{HN}-\mathrm{I}$ & KF429254 & China & ORFs & 2013 \\
\hline HN-G & KF429252 & China & ORFs & 2012 \\
\hline JT_NS3 & JN968975 & China & ORFs & 2010 \\
\hline 33790005_6a & GQ884043 & Europe & ORFs & 2006 \\
\hline 40090005_1h & GQ884045 & USA & ORFs & 2006 \\
\hline J-PPV & KF742500 & China & ORFs & 2013 \\
\hline HN-K & KF429255 & China & ORFs & 2013 \\
\hline 21620005_1h & GQ884035 & Europe & ORFs & 2005 \\
\hline N & HM989009 & China & ORFs & 1989 \\
\hline China & AY583318 & China & ORFs & 2004 \\
\hline 32360005_1f & GQ884040 & Europe & ORFs & 2005 \\
\hline VRI-1 & AY390557 & South Korea & ORFs & 2003 \\
\hline PPV/Tornau/1/02 & AY684869 & Germany & ORFs & 2002 \\
\hline VaccinevirusIDT & AY684872 & Germany & ORFs & 1964 \\
\hline N108 & MH566237 & South Korea & ORFs & 2018 \\
\hline HNZK-1 & KJ201928 & China & ORFs & 2007 \\
\hline HNAY & KJ201927 & China & ORFs & 2009 \\
\hline 16WS & KM268633 & China & ORFs & 2013 \\
\hline TJ & KX233726 & China & ORFs & 2015 \\
\hline Nanjing200801 & FJ822038 & China & ORFs & 2008 \\
\hline N91 & MH817778 & South Korea & ORFs & 2018 \\
\hline N2 & MH817779 & South Korea & ORFs & 2018 \\
\hline NJ & AY686601 & China & NS1 & - \\
\hline NJ & AY686602 & China & VP2 & - \\
\hline
\end{tabular}

Explanation: - Not identified 
remaining supernatant was stored at $-80^{\circ} \mathrm{C}$ until further use. The viral DNA obtained was stored at $-20^{\circ} \mathrm{C}$ until required.

Primers were designed according to the reported genomic sequences of PPV retrieved from GenBank of the National Center for Biotechnology Information and analyzed with the Lasergene package (DNAStar Inc., Madison,WI, USA). A pair of primers (PPV-1F/PPV-1R), located in the conserved region of the genome, was designed to amplify a 502 bp fragment of PPV DNA (Tab. 2). PCR was performed under the following conditions: initial denaturation at $94^{\circ} \mathrm{C}$ for $5 \mathrm{~min}$, 35 cycles at $94^{\circ} \mathrm{C}$ for $30 \mathrm{~s}, 55^{\circ} \mathrm{C}$ for $30 \mathrm{~s}$, and $72^{\circ} \mathrm{C}$ for $20 \mathrm{~s}$, followed by a final extension at $72^{\circ} \mathrm{C}$ for $7 \mathrm{~min}$. The PCR products were analyzed by agarose gel electrophoresis, and the target bands were excised.

Virus isolation and genome sequencing. The supernatants identified as PPV-positive by PCR detection were then passed through sterile $0.22 \mu \mathrm{m}$ filters (Pall Corp., Port Washington, NY, USA), diluted 1 : 10 in Dulbecco's modified Eagle medium (DMEM) containing 1\% penicil$\mathrm{lin} /$ streptomycin, and incubated with monolayers of swine testicular (ST) cells in 24-well culture plates (Corning, Corning, NY, USA) for $1 \mathrm{~h}$ at $37^{\circ} \mathrm{C}$ in a $5 \% \mathrm{CO}_{2}$ environment. Fresh DMEM/2\% newborn calf serum (Invitrogen, Waltham, MA, USA) $/ 1 \%$ penicillin/streptomycin was then added to the culture, and the incubation was continued for 4 days. The cultures were freeze-thawed three times and centrifuged at $4,000 \times g$ for $5 \mathrm{~min}$. The clarified supernatants were then repassaged into fresh ST cells, and the culture supernatants were collected after three passages and stored at $-80^{\circ} \mathrm{C}$ until required.

The viral DNA was extracted from the supernatants according to the manufacturer's instructions (Tiandz Inc., Beijing, China) and used for PCR amplification with pfu DNA Polymerase (Tsingke, China). Six primer sets (Tab. 2) were designed to amplify overlapping regions of the ORFs of each identified PPV isolate on the basis of published genomic sequences of PPV reference isolates (Tab. 3). The PCR products were purified by agarose gel electrophoresis and then sequenced at Tsingke (China).

Sequence and statistical analysis. The sequences obtained were analyzed by DNAMAN 7.0 (Lynnon Corporation). Reference PPV genomes were downloaded from GenBank for alignments and phylogenetic analysis (Tab. 3). Phylogenetic analyses were carried out by MEGA 6.06 using the maximum-likelihood method (23).

Differences in prevalence between sample types were investigated by chi-square tests from SPSS software version 19.0 (IBM Inc., Chicago, IL, USA). A $P$ value of less than 0.05 was considered statistically significant.

\section{Results and discussion}

Prevalence of PPV. PPV was detected in 84 of the 1093 samples, representing an overall PPV-positivity rate of $7.69 \%$. Among all sample types, PPV was most frequently detected in the lung samples, with a prevalence of $22.90 \%(71 / 310)$. PPV was also detected in the fecal swabs $(2.54 \%, 5 / 197)$, nasal swabs $(2.03 \%$, $4 / 197)$, and serum samples $(1.03 \%, 4 / 389)$, but with significantly $(\mathrm{P}<0.01)$ lower rates than those for the lungs (Tab. 1).

Virus isolation and identification. Five of the 84 PPV-positive samples identified by PCR showed a cytopathic effect when incubated with ST cells for 4 days, characterized by ramification, rounding, and detachment of the cells. The approximately complete genomes of these five PPV isolates, tentatively named PPV-HuN1-5, were sequenced and submitted to GenBank (accession nos. MH183294-MH183298). The entire coding sequences of the five PPV-HuNs contain 4258 nucleotides.

Molecular and phylogenetic analysis. Alignment of the NS1 sequences of PPV-HuN1-5 revealed high nucleotide and amino acid identities in the range of 99.6-100\% and 99.5-100\%, respectively. Homology comparison of VP2 genes revealed complete nucleotide and deduced amino acid sequence identities. Moreover, seven amino acid substitutions were identified in the VP2 sequences of the five PPV-HuNs (Q228E, N341Y, D378G, H383Q, A414S, E419Q, and P436T) compared with the VP2 of the Chinese vaccine strain PPV-NJ (Fig. 1).

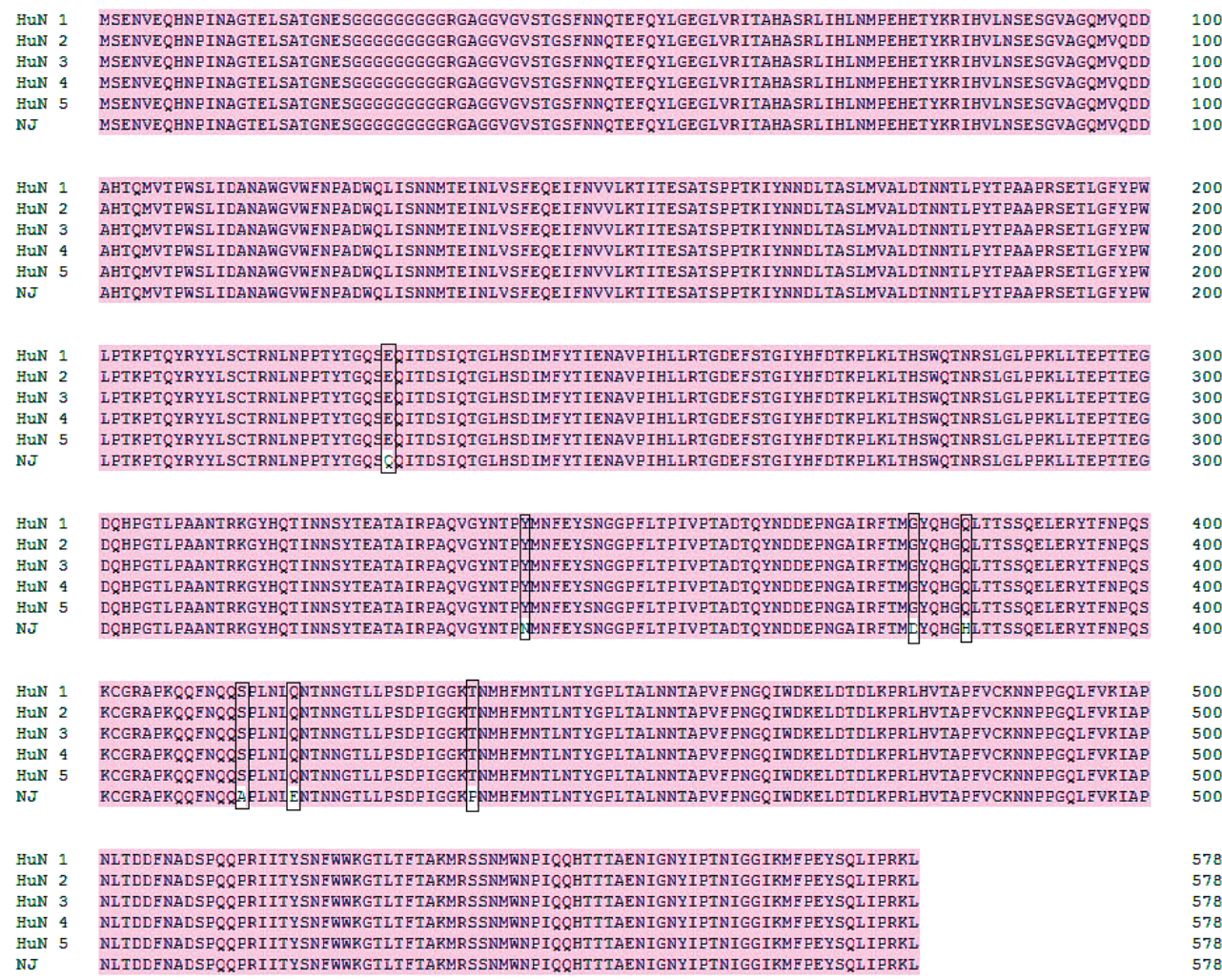

Fig. 1. Alignment of the VP2 amino acid sequences of PPV-HuNs with the Chinese vaccine strain PPV-NJ. Sequences in the boxes show the amino acid substitutions. 
Tab. 4. Homology comparison of porcine parvovirus complete ORFs genes

\begin{tabular}{|c|c|c|c|c|c|}
\hline \multirow{2}{*}{ Strain ${ }^{\mathrm{a}}$} & \multicolumn{5}{|c|}{ \% nucleotide identities: } \\
\hline & HuN1 & HuN2 & HuN3 & HuN4 & HuN5 \\
\hline HuN1 & & & & & \\
\hline HuN2 & 100.0 & & & & \\
\hline HuN3 & 99.9 & 99.9 & & & \\
\hline HuN4 & 99.9 & 99.9 & 99.8 & & \\
\hline HuN5 & 99.9 & 99.9 & 99.9 & 99.8 & \\
\hline 32350005_3h & 99.9 & 99.9 & 99.9 & 99.8 & 99.9 \\
\hline 32260005erh_1h & 99.7 & 99.7 & 99.8 & 99.6 & 99.7 \\
\hline 2012 & 99.7 & 99.7 & 99.7 & 99.6 & 99.7 \\
\hline 14a & 99.7 & 99.7 & 99.7 & 99.6 & 99.7 \\
\hline 8a & 99.6 & 99.6 & 99.6 & 99.5 & 99.6 \\
\hline $7 a$ & 99.6 & 99.6 & 99.6 & 99.5 & 99.6 \\
\hline 33800005_1g & 99.6 & 99.6 & 99.6 & 99.5 & 99.6 \\
\hline JY & 99.6 & 99.6 & 99.6 & 99.5 & 99.6 \\
\hline 32260005_1d & 99.4 & 99.4 & 99.5 & 99.3 & 99.4 \\
\hline $2 f$ & 99.4 & 99.4 & 99.4 & 99.3 & 99.4 \\
\hline 33760005_3a & 99.3 & 99.3 & 99.3 & 99.2 & 99.3 \\
\hline 693a & 99.3 & 99.3 & 99.3 & 99.2 & 99.3 \\
\hline 33780005_2g & 99.2 & 99.2 & 99.2 & 99.1 & 99.2 \\
\hline Challenge & 99.2 & 99.2 & 99.2 & 99.1 & 99.2 \\
\hline Kresse & 99.2 & 99.2 & 99.2 & 99.1 & 99.2 \\
\hline 40570005_4e & 99.1 & 99.1 & 99.2 & 99.0 & 99.1 \\
\hline HN-2011 & 99.2 & 99.2 & 99.2 & 99.1 & 99.2 \\
\hline ZJ & 99.2 & 99.2 & 99.2 & 99.1 & 99.2 \\
\hline HNLY201301 & 99.1 & 99.1 & 99.2 & 99.0 & 99.1 \\
\hline NADL-2 & 99.1 & 99.1 & 99.2 & 99.0 & 99.1 \\
\hline YL & 99.1 & 99.1 & 99.1 & 99.0 & 99.1 \\
\hline T142_Korea & 99.1 & 99.1 & 99.1 & 99.0 & 99.1 \\
\hline LZ & 99.1 & 99.1 & 99.1 & 99.0 & 99.1 \\
\hline BQ & 99.1 & 99.1 & 99.1 & 99.0 & 99.1 \\
\hline HN-H & 99.1 & 99.1 & 99.1 & 99.0 & 99.1 \\
\hline PPV2010 & 99.1 & 99.1 & 99.1 & 99.0 & 99.1 \\
\hline SR-1 & 99.1 & 99.1 & 99.1 & 99.0 & 99.1 \\
\hline GD2013 & 99.0 & 99.0 & 99.1 & 98.9 & 99.0 \\
\hline $\mathrm{HN}-\mathrm{I}$ & 99.0 & 99.0 & 99.0 & 98.9 & 99.0 \\
\hline HN-G & 99.0 & 99.0 & 99.0 & 98.9 & 99.0 \\
\hline JT_NS3 & 99.0 & 99.0 & 99.0 & 98.9 & 99.0 \\
\hline 33790005_6a & 99.0 & 99.0 & 99.0 & 98.9 & 99.0 \\
\hline 40090005_1h & 99.0 & 99.0 & 99.0 & 98.9 & 99.0 \\
\hline J-PPV & 99.0 & 99.0 & 99.0 & 98.9 & 99.0 \\
\hline HN-K & 99.0 & 99.0 & 99.0 & 98.9 & 99.0 \\
\hline 21620005_1h & 99.0 & 99.0 & 99.0 & 98.9 & 99.0 \\
\hline $\mathbf{N}$ & 99.0 & 99.0 & 99.0 & 98.9 & 99.0 \\
\hline China & 99.0 & 99.0 & 99.0 & 98.9 & 99.0 \\
\hline 32360005_1f & 98.9 & 98.9 & 99.0 & 98.9 & 98.9 \\
\hline VRI-1 & 99.0 & 99.0 & 99.0 & 98.9 & 99.0 \\
\hline 2 & 98.9 & 98.9 & 98.9 & 98.8 & 98.9 \\
\hline VaccinevirusIDT & 98.9 & 98.9 & 98.9 & 98.8 & 98.9 \\
\hline N108 & 98.8 & 98.8 & 98.8 & 98.7 & 98.8 \\
\hline HNZK-1 & 98.8 & 98.8 & 98.8 & 98.7 & 98.8 \\
\hline HNAY & 98.8 & 98.8 & 98.8 & 98.7 & 98.8 \\
\hline 16WS & 98.8 & 98.8 & 98.8 & 98.7 & 98.8 \\
\hline TJ & 98.7 & 98.7 & 98.8 & 98.6 & 98.7 \\
\hline Nanjing200801 & 98.7 & 98.7 & 98.7 & 98.6 & 98.7 \\
\hline N91 & 98.6 & 98.6 & 98.6 & 98.5 & 98.6 \\
\hline N2 & 98.4 & 98.4 & 98.4 & 98.3 & 98.4 \\
\hline
\end{tabular}

Explanation: a - Details of reference strains in Supplementary Table 2.

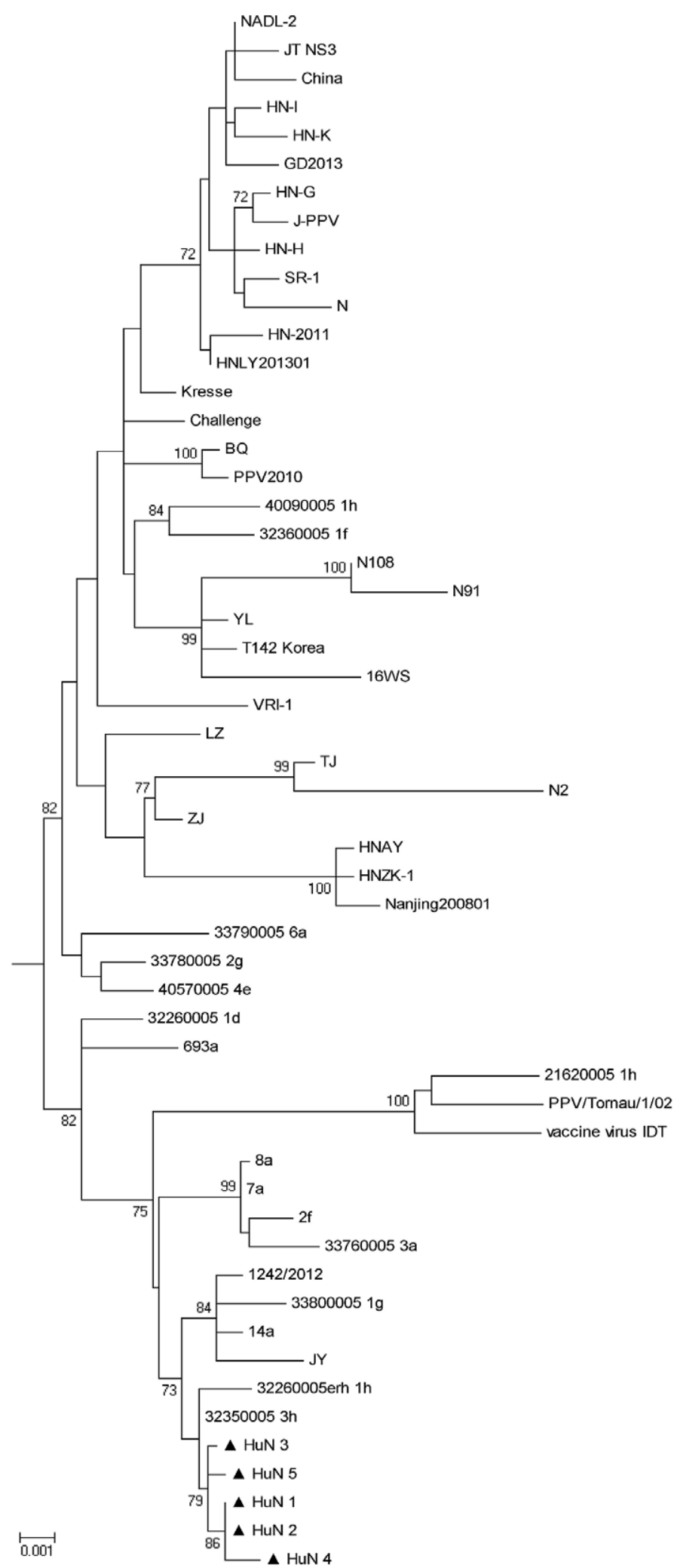

Fig. 2. Phylogenetic tree based on the complete ORFs sequences of all available PPV strains. The Hasegawa-Kishino-Yano model with the proportion of invariant sites and gammadistributed rate heterogeneity was used to construct the Maximum Likelihood tree with 1000 bootstrap replications to test the phylogeny. The percentage of replicates in which the associated virus clustered together in the bootstrap test $(1000$ replicates) is shown next to the branches in each tree (only values $>\mathbf{7 0} \%$ are shown). The scale bar indicates nucleotide substitutions per site. The strains isolated in the present study are identified by $\Delta$. 
Homology comparison of the complete ORF sequences of PPV-HuN1-5 with all other reference strains in GenBank showed the closest relationship to the PPV-32350005 3h sequence (GQ884039) from Europe, with an average nucleotide homology of 99.9\% (Tab. 4). Moreover, in the phylogenetic analysis of complete ORFs, the five PPV-HuNs clustered in the same clade and showed a close relationship to PPV strains from Europe (e.g., 32350005 3h, 32260005erh_1h, 33800005_1g, 1242/2012, and $\overline{14 a)}$ and China (JY) (Fig. 2).

Since PPV was first recognized at the end of the 1960 s, it has been discovered in pigs worldwide, being one of the most common and important infectious agents of infertility and causing economic losses to the swine industry (15). PPV outbreaks have been reported in several countries $(8,11)$. In general, PPV infection during the first half of pregnancy can lead to the reproductive failure of sows, but does not cause clinical symptoms in non-pregnant adult pigs or piglets (15). According to studies on abortion cases with clinical and experimental infection of PPV field viruses, PPV antigens can be detected in multiple tissues, including the heart, liver, kidney, duodenum, thymus, lung, spleen, brain, and lymph nodes of fetuses, as well as in the placenta and uterus of sows $(11,24)$. However, information on the epidemiology and tissue tropism of PPV infection in healthy non-pregnant adult pigs is scarce. In the present study, we detected a high prevalence of PPV in the lungs of apparently healthy fattening pigs, whereas the detection rates in fecal swabs, nasal swabs, and serum samples were low. This finding may indicate tissue tropism to the lungs. However, further studies with more samples and sample types are needed to confirm these findings to clarify the tissue tropism and distribution of PPV in healthy non-pregnant adult pigs.

Although an inactivated PPV vaccine has been widely used in China, only sows are typically inoculated in most herds. In general, the maternal antibodies produced from the vaccine can offer passive protection to the piglets until 9-22 weeks of age (7). Therefore, the finding of this high prevalence of PPV in slaughtered pigs which were no longer passively protected from milk but not yet vaccinated was not unexpected. Moreover, the rare presence of PPV in fecal swabs, nasal swabs, and serum might indicate short-term shedding of the virus and viremia in healthy fattening pigs after PPV infection. Nevertheless, as fattening pigs represent the largest population in the herd, such longterm PPV carriers would pose a high risk of spread and a challenge for PPV disease prevention and control.

PPV was previously considered to have a conservative genome (12). However, recent studies based on systematic analyses of the genetic variation and evolution of the virus indicated high mutation rates (approximately $10^{-4}$ ) in the VP genes, with a more moderate evolutionary rate for NS genes (approximately $\left.10^{-5}\right)(18,21)$. Although several studies focused on the genetic diversity of PPV field strains from both domestic pigs and wild boars, the classification of PPV subgroups remains ambiguous $(3,16,18)$. Moreover, no obvious correlation has been observed between clustering from phylogeny and geographical distribution or the virulence status of PPV isolates (15). The present findings fit with this general pattern, as the five PPV-HuN isolates obtained in this study from Hunan showed a relatively closer genetic relationship with each other, and phylogenetic analysis of complete ORFs demonstrated a close relationship of these isolates with most of the European PPV strains.

Inactivated vaccines have been widely used and have conferred good protection against PPV disease for a long time. However, recent studies conducted in Germany indicated that although these inactivated vaccines are effective against homologous infections, they could not prevent infection and virus shedding after challenge with the antigenically heterologous 27 a strain $(9,26)$. The determinants of PPV virulence have not yet been identified, but several studies suggest that the amino acid residue substitutions in accessible regions on the capsid surface, especially the positions of linear epitopes on VP2, play an important role in the pathogenicity of PPV in vivo $(10,15,22)$. Indeed, we identified seven amino acid substitutions in the coding region of VP2 between PPV-HuN1-5 and the Chinese vaccine strain PPV-NJ. Three of these amino acid positions $(378,383$, and 436$)$ are considered to be responsible for host tropism in tissue culture cells (2). A three-dimensional model of PPV VP2 predicted amino acids 378 and 383 to be located close to the 2 -fold axis on the surface of the virus capsid, and position 436 is located within the 3 -fold spike center of the capsid subunit, indicating that these sites may be involved in the immune response and virulence (22). However, further investigation is needed to clarify the precise influence of these amino acid variations on the capsid structure and immune response.

\section{References}

1. Afolabi K. O., Iweriebor B. C., Obi L. C., Okoh A. I.: Prevalence of porcine parvoviruses in some South African swine herds with background of porcine circovirus type 2 infection. Acta. Trop. 2019, 190, 37-44.

2. Bergeron J., Hebert B., Tijssen P.: Genome organization of the Kresse strain of porcine parvovirus: identification of the allotropic determinant and comparison with those of NADL-2 and field isolates. J. Virol. 1996, 70, 2508-2515.

3. Cadar D., Dan A., Tombacz K., Lorincz M., Kiss T., Becskei Z., Spinu M., Tuboly T., Csagola A.: Phylogeny and evolutionary genetics of porcine parvovirus in wild boars. Infect. Genet. Evol. 2012, 12, 1163-1171.

4. Coackley W., Smith V. W.: Porcine parvoviruses in Western Australia. Aust. Vet. J. 1972, 48, 536.

5. Cotmore S. F., Agbandje-McKenna M., Canuti M., Chiorini J. A., Eis-Hubinger A. M., Hughes J., Mietzsch M., Modha S., Ogliastro M., Penzes J. J., Pintel D. J., Qiu J., Soderlund-Venermo M., Tattersall P., Tijssen P., Ictv R. C.: ICTV Virus Taxonomy Profile: Parvoviridae. J. Gen. Virol. 2019, 100, 367-368.

6. Csagola A., Lorincz M., Cadar D., Tombacz K., Biksi I., Tuboly T.: Detection, prevalence and analysis of emerging porcine parvovirus infections. Arch. Virol. 2012, 157, 1003-1010.

7.Etoh M., Morishita E., Ochiai M., Watanabe Y.: Transitional antibodies and spontaneous infection in the porcine (Sus scrofa) parvo viral infections. Japanese Journal of Swine Husbandry Research, Japan 1979, 16, 237-239. 
8. Hao X., Lu Z., Sun P., Fu Y., Cao Y., Li P., Bai X., Bao H., Xie B., Chen Y., Li D., Liu $Z$.: Phylogenetic analysis of porcine parvoviruses from swine samples in China. Virol. J. 2011, 8, 320.

9. Jozwik A., Manteufel J., Selbitz H. J., Truyen U.: Vaccination against porcine parvovirus protects against disease, but does not prevent infection and virus shedding after challenge infection with a heterologous virus strain. J. Gen. Virol. 2009, 90, 2437-2441.

10. Kamstrup S., Langeveld J., Botner A., Nielsen J., Schaaper W. M., Boshuizen R. S., Casal J. I., Hojrup P., Vela C., Meloen R., Dalsgaard K.: Mapping the antigenic structure of porcine parvovirus at the level of peptides. Virus. Res. 1998, 53, 163-173

11. Kaur A., Mahajan V., Leishangthem G. D., Singh N. D., Bhat P., Banga H. S., Filia G.: Epidemiological and immunopathological studies on Porcine parvovirus infection in Punjab. Vet. World. 2016, 9, 827-831.

12. Lopez-Bueno A., Villarreal L. P., Almendral J. M.: Parvovirus variation for disease: a difference with RNA viruses? Curr. Top. Microbiol. Immunol. 2006 299, 349-370.

13. Mayr A., Mahnel H.: Cultivation of hog cholera virus in pig kidney cultures with cytopathogenic effect. Zentralbl. Bakteriol. Orig. 1964, 195, 157-166.

14. Mengeling W. L., Lager K. M., Vorwald A. C.: The effect of porcine parvovirus and porcine reproductive and respiratory syndrome virus on porcine reproductive performance. Anim. Reprod. Sci. 2000, 60-61, 199-210.

15. Meszaros I., Olasz F., Csagola A., Tijssen P., Zadori Z.: Biology of Porcine Parvovirus (Ungulate parvovirus 1). Viruses 2017, 9, 393.

16. Oh W. T., Kim R. Y., Nguyen V. G., Chung H. C., Park B. K.: Perspectives on the Evolution of Porcine Parvovirus. Viruses 2017, 9, 196.

17. Opriessnig T., Xiao C. T., Gerber P. F., Halbur P. G.: Identification of recently described porcine parvoviruses in archived North American samples from 1996 and association with porcine circovirus associated disease. Vet. Microbiol. $2014,173,9-16$
18. Ren X., Tao Y., Cui J., Suo S., Cong Y., Tijssen P.: Phylogeny and evolution of porcine parvovirus. Virus. Res. 2013, 178, 392-397.

19. Shangjin C., Cortey M., Segales J.: Phylogeny and evolution of the NS1 and VP1/VP2 gene sequences from porcine parvovirus. Virus. Res. 2009, 140, 209-215.

20. Simpson A. A., Hebert B., Sullivan G. M., Parrish C. R., Zadori Z., Tijssen P., Rossmann $M$. G.: The structure of porcine parvovirus: comparison with related viruses. J. Mol. Biol. 2002, 315, 1189-1198.

21. Streck A. F., Bonatto S. L., Homeier T., Souza C. K., Goncalves K. R., Gava D. Canal C. W., Truyen $U$. : High rate of viral evolution in the capsid protein of porcine parvovirus. J. Gen. Virol. 2011, 92, 2628-2636.

22. Streck A. F., Canal C. W., Truyen U.: Molecular epidemiology and evolution of porcine parvoviruses. Infect. Genet. Evol. 2015, 36, 300-306.

23. Tamura K., Stecher G., Peterson D., Filipski A., Kumar S.: MEGA6: Molecular Evolutionary Genetics Analysis version 6.0. Mol. Biol. Evol. 2013, 30, 2725 -2729 .

24. Wilhelm S., Zeeuw E. J., Selbitz H. J., Truyen U.: Tissue distribution of two field isolates and two vaccine strains of porcine parvovirus in foetal organs after experimental infection of pregnant sows as determined by real-time PCR. J. Vet. Med. B. Infect. Dis. Vet. Public. Health. 2005, 52, 323-326.

25. Zadori Z., Szelei J., Tijssen P.: SAT: a late NS protein of porcine parvovirus. J. Virol. 2005, 79, 13129-13138.

26.Zeeuw E. J., Leinecker N., Herwig V., Selbitz H. J., Truyen U.: Study of the virulence and cross-neutralization capability of recent porcine parvovirus field isolates and vaccine viruses in experimentally infected pregnant gilts. J. Gen. Virol. 2007, 88, 420-427.

Corresponding author: Xinglong Yu, Prof. Dr., College of Veterinary Medicine, Hunan Agricultural University, Changsha, Hunan 410128, China; e-mail: xlyu999@126.com 